\title{
Scientists in a time of COVID-19
}

\author{
The COVID-19 pandemic has been cutting us all off from the social aspects of human community for several \\ months. High time we checked in on our plant science colleagues to see how they are faring.
}

M aeli Melotto at the University of California, Davis, told us "my lab has been closed since 19 March", and when we asked how COVID19 was affecting her research, she said "my whole research team is working on writing (projects and manuscripts), data analysis and/or remote training".

This story is echoed by the researchers we have talked to recently. At the RIKEN Center for Sustainable Resource Science, Japan, all of Keiko Sugimoto and her team's ongoing experiments have had to stop, as "all labs in our institute have shut down since early April". At the VIB-University of Ghent Center for Plant Systems Biology, Belgium, "almost all wet lab activities have come to a full stop and teleworking from the home office has become a standard", according to Moritz Nowack.

While it may be possible for some researchers to wait out this hiatus, there is an immediate risk to researchers approaching the end of their grants or contracts. Experiments crucial for completing publications needed to secure their next posts have had to be put on-hold. Researchers in the early stages of a project may also be in danger of falling behind. All experimentalists may need additional funding to recover such lost time. As a result, Sugimoto sees signs of "some early-career researchers quickly losing motivation for science".

Routine maintenance of experimental plants, at least, is little affected. In some institutes, dedicated staff, following social distancing, take care of plants growing in greenhouses. Elsewhere, volunteers are allowed to enter labs to maintain precious experimental materials. "We have one member of the lab to water plants for the whole group", says Hao Yu, a Principle Investigator at the National University of Singapore.

Others have found ways to adapt their expertise. "Three people in my lab have started a new project to express coronavirus proteins in tobacco", says David Jackson, whose lab is situated at the Cold Spring Harbor Laboratory, Long Island, New York. "We hope to provide these materials to our collaborators at Columbia University for their research on immune responses in patients", he continues, "and at least the new project can keep us involved and help tackle this important problem".
But for most researchers, remote working is the only option. Internal lab meetings and journal clubs are fairly easy to conduct with virtual meeting tools, such as Zoom and GoToMeeting. Researchers may even be meeting more often now since joint lab meetings, even across continents, have become, if anything, more feasible than before.

Working from home creates new challenges for work-life balance. Yu feels that "the day is getting longer, as I have been working throughout the day in the same chair". This 'long day' feeling is shared by many, as online meetings, teaching and research activities disrupt the boundaries between work and home. Researchers with children have the added challenge of balancing full-time work in their home office and full-time parenting while schools are closed. "This is certainly not the time to sit down and finish pending manuscripts", says Nowack, who continues by stating "for all young parents, this situation is emotionally rather taxing, as neither the job nor the family can be given the attention they really deserve".

Restaurants and cleaning services are stunted by the pandemic, giving researchers increased cooking and household duties. On average, women carry more childcare and housework responsibilities than their male counterparts. Therefore, female researchers are likely to suffer more from the crisis than their male colleagues. Recent analyses suggest that gender disparities have become more severe under the emergent conditions (Nature 581, 365-366; 2020). The number of female authors on preprints posted to bioRxiv in March to April 2020 has grown slower than for male authors (https://bit. ly/36VjayZ), and new projects registered by female principal investigators is a smaller proportion of total research projects than that before the COVID-19 outbreak (https:// go.nature.com/2XN8YVi).

And yet there are upsides to this crisis. "We are getting very used to interacting with each other online, which allows short and very productive exchanges of ideas", says Lucia Colombo from the University of Milan, Italy. Zhaosheng Kong, at the Institute of Microbiology, Chinese Academy of Sciences, has found that "my students never stop learning during the pandemic. The various webinars and other forms of online science communications provide new opportunities for them to learn and develop a good sense of science". But a return to regular activities is now becoming possible in both China and Italy. Colombo says that "the situation is getting much better after lockdown. We are now allowed to work in the lab again and we are working in shifts for seven days a week to keep the numbers of people working in the lab as low as possible".

Without time wasted on commuting, many are spending more time with close family. "My little daughter is very excited because I spend more time playing with her, and my wife is also happy because I cook more frequently", says Kong. Sugimoto agrees, saying "the kids now see what I do more closely and seem to appreciate both sides of me, being a scientist and mother, much more". Nowack also has deep feelings: "my daughter could only crawl before the lockdown and now is running around in the garden. And I deeply enjoy watching the blue sky without contrails as a daily reminder of what we are doing to our planet under normal circumstances". In addition, Kong finds that a slow-paced life gives "more time to conceive long-term projects".

However, conferences and meetings scheduled during 2020 are being cancelled, postponed or moved to virtual platforms. "I do feel in a way that we are closer together in the world", says Sugimoto. "I am exposed to new people out of my usual routine and I am continuing discussions with some of my webinar audience". Colombo agrees but misses physical meetings, as an online event "never replaces the social contact, like eating or drinking together, which are often the occasions where new ideas and collaborations are born". Yu adds "sometimes it is just nice to get out of the office and enjoy science communication in another part of the world".

This pandemic will be long-lasting, at least until a treatment or vaccine is developed. Even so, the plant researchers we talk to are optimistic. As Yu puts it, scientists "might find a better reason to dedicate their life to plant research, because food production is extremely important under such kinds of deadly pandemics".

Published online: 15 June 2020 https://doi.org/10.1038/s41477-020-0714-3 